\title{
Estágio reprodutivo, histologia e morfometria sazonal do testículo de Dermanura cinerea (Chiroptera: Phyllostomidae) em fragmento de Mata Atlântica no Litoral Sul de Pernambuco, Brasil
}

\author{
Nivaldo B. de Lima Junior' (D), Maria J. G. Arandas (D), Fabricya R. da Silva' (1), Diêgo V. da Silva² (D), Álvaro \\ A. C. Teixeira' (1), Francisco C. A. de Aguiar Júnior² (D), José E. Garcia² (i) \& Katharine R. P. dos Santos ${ }^{2}$ (i)
}

\footnotetext{
1. Universidade Federal Rural de Pernambuco (UFRPE), Departamento de Morfologia e Fisiologia Animal (DMFA), Rua Dom Manoel de Medeiros s/n, Dois Irmãos, 52171-900 Recife, PE, Brasil (nivaldobernardo@hotmail.com)

2.Universidade Federal de Pernambuco (UFPE), Centro Acadêmico de Vitória (CAV), Núcleo de Biologia, Rua Alto do Reservatório s/n, Bela Vista, 55608-680 Vitória de Santo Antão, PE, Brasil.
}

\begin{abstract}
Reproductive stage, histology and seasonal morphometry of the testes of Dermanura cinerea (Chiroptera: Phyllostomidae) in Atlantic Forest fragment on the South Coast of Pernambuco, Brazil. This study analyzed the reproductive stage, histology and morphometry of the testes of Dermanura cinerea (Gervais, 1856) in Atlantic Forest fragment on the South Coast of Pernambuco. Specimens were captured by mist net, and collections occurred monthly over two consecutive nights for 18 months. The meteorological data were grouped in rainy and dry months. Eighteen adult specimens were used, which were classified in males with descendant testes and with testes no descendant. For the histological analysis, the testes were collected, fixed and processed following the routine histological technique. The slides obtained were stained by Hematoxylin-Eosin. In the morphometric analysis the area of occupation of the tubular and intertubular compartment were measured, the number of Leydig, Sertoli, spermatocytes and elongated spermatids were quantified. The morphometric data were submitted to statistical analysis. Dermanura cinerea presented higher sperm production activity in dry months and higher hormone production in rainy months. This information is also related to the reproductive pattern of the females and to the increase in rainfall indexes, since it is a determinant factor in the mediation of the food availability of the frugivorous species, such as $D$. cinerea in the Atlantic Forest area of Pernambuco.
\end{abstract}

KEYWORDS. Bat, gonadal histomorphometry, morphology, Northeast, seasonality.

RESUMO. Esse estudo analisou o estágio reprodutivo, a histologia e a morfometria dos testículos de Dermanura cinerea (Gervais, 1856) em fragmento de Mata Atlântica no Litoral Sul de Pernambuco. Os espécimes foram capturados por redes de neblina e as coletas ocorreram mensalmente, ao longo de duas noites consecutivas, durante 18 meses. Os dados meteorológicos foram agrupados em meses chuvosos e secos. Foram utilizados 18 espécimes adultos, os quais foram classificados em machos com testículos descendentes e não descendentes. Para as análises histológicas, os testículos foram coletados, fixados e processados seguindo a técnica histológica de rotina. As lâminas obtidas foram coradas por Hematoxilina-Eosina. Nas análises morfométricas foram mensuradas a área de ocupação do compartimento tubular e intertubular, quantificados o número de células de Leydig, de Sertoli, de espermatócitos e de espermátides alongadas. Os dados morfométricos foram submetidos às análises estatísticas. Dermanura cinerea apresentou maior atividade de produção espermática em meses secos e maior produção hormonal em meses chuvosos. Essas informações estão relacionadas também com o padrão reprodutivo das fêmeas e com a elevação nos índices pluviométricos, já que é um fator determinante na mediação da disponibilidade alimentar das espécies frugívoras, como D. cinerea em área de Mata Atlântica de Pernambuco.

PALAVRAS-CHAVE. Morcego, Histomorfometria gonadal, morfologia, Nordeste, sazonalidade.

A biologia reprodutiva de Chiroptera ainda é uma área pouco investigada, sobretudo, no Brasil. Na Região Neotropical, a reprodução desse grupo pode passar por alterações ao longo do ano, associadas a fatores como disponibilidade alimentar, temperatura, precipitação e fotoperíodo. As diferentes espécies de morcegos dessa região se reproduzem em períodos em que as condições ambientais são mais favoráveis; assim, uma mesma espécie pode apresentar padrão ou estratégia reprodutiva diferente, dependendo do período considerado e da sua distribuição (FABiÁn \& MARQUES, 1989; Crichton \& KRUtZsch, 2000;
Neuweiler, 2000; ZortéA, 2003; Mello et al., 2004; BEGUELINI et al., 2009).

As investigações acerca da reprodução dos morcegos afirmavam que, em relação à posição anatômica das gônadas, os testículos situados na região escrotal (descendentes) indicavam que o animal estava sexualmente ativo, ao passo que os localizados na região abdominal (não descendentes) demonstravam uma inatividade sexual (BREDT et al., 1999; ZORTÉA, 2003; GOMES \& UIEDA, 2004; ORTÊNCIO FILHO et al., 2007). Contudo, Lima JUNIOR et al. (2014) comprovou, por meio da histomorfometria testicular, que avaliar a reprodução 
com base apenas nessas constatações, leva a inferências limitadas e por vezes errôneas, pois verificaram que os espécimes de Phyllostomus discolor (Wagner, 1843) mesmo com testículos não descendentes, apresentaram atividade espermatogênica.

A histologia e a morfometria dos testículos são parâmetros que contribuem para mensurar de forma mais precisa as variações nos elementos que compõe essas gônadas, como os tipos celulares e os compartimentos intertubular e tubular. Por meio de medidas da área e diâmetro dos túbulos seminíferos, bem como da altura do seu epitélio e da quantificação dos tipos celulares é possível avaliar a eficiência espermatogênica da espécie e sua capacidade reprodutiva (FrançA \& Russell, 1998; Morais et al., 2013a,c).

Dermanura cinerea (Gervais, 1856) pertence à família Phyllostomidae e subfamília Stenodermatinae, anteriormente era conhecida como Artibeus cinereus (Gervais, 1856) (REDONDO et al., 2008; SolARI et al., 2009). Sua ocorrência já foi relatada em quatro regiões brasileiras: Norte, Nordeste, Sudeste e Sul, sobretudo, em fragmentos florestais da Mata Atlântica, bem como em matas ciliares primárias (SCULTORI et al., 2009; ReIS et al., 2011). Os estudos sobre a reprodução dessa espécie no Brasil são limitados (RocHA et al., 2010). Desse modo, investigações sobre histomorfometria testicular, bem como sobre qual o padrão e estratégia reprodutiva desenvolvida por essa espécie não existem no estado de Pernambuco.

Perante essas investigações, esse trabalho teve como objetivo analisar o estágio reprodutivo, a histologia e a morfometria testicular de $D$. cinerea coletados em área de Mata Atlântica situada no litoral sul do estado de Pernambuco, em meses chuvosos e secos do ciclo reprodutivo anual.

\section{MATERIAL E MÉTODOS}

Área de estudo e exemplares. Os animais foram coletados, em fragmento de Mata Atlântica (844'19.9'S $35^{\circ} 10$ '19.1"W) do litoral sul de Pernambuco, com a instalação de dez redes de neblina (12 $\mathrm{m}$ de comprimento $\mathrm{x}$ $3 \mathrm{~m}$ de altura) em cada noite de captura. As coletas ocorreram mensalmente, ao longo de duas noites consecutivas, durante 18 meses (junho/2014 a novembro/2015). Em cada noite de captura, as redes eram estendidas às $17 \mathrm{~h} 00 \mathrm{~min}$ e ficavam abertas até às $00 \mathrm{~h} 00 \mathrm{~min}$, sendo verificadas continuamente em intervalos de 15 minutos. O esforço total de captura foi de $90720 \mathrm{~m}^{2}$.h. (Straube \& Bianconi, 2002). Foram capturados 18 espécimes machos adultos de Dermanura cinerea.

Esse estudo obteve a aprovação da Comissão de Ética no Uso de Animais (CEUA), da Universidade Federal de Pernambuco - UFPE (protocolo no 23076.037360/2014 - 92).

Os espécimes coletados encontram-se armazenados temporariamente no Laboratório de Biotecnologia e Fármacos da Universidade Federal de Pernambuco (UFPE), campus de Vitória de Santo Antão (CAV) e serão depositados na coleção da UFPE campus Recife.

Dados meteorológicos. As informações meteorológicas foram fornecidas pelo Instituto Nacional de
Meteorologia - INMET/Seção de Observação e Meteorologia Aplicada - SEOMA. Com base nessas informações, e considerando as variações nas precipitações pluviométricas, as análises dos aspectos reprodutivos de $D$. cinerea foram agrupadas em dois períodos de nove meses cada.

Esse agrupamento foi realizado seguindo um padrão do que é determinado para o período seco e chuvoso na localidade de estudo. Para isso foi calculada a mediana (130, 95 milímetros) dos valores das precipitações mensais, de modo que a partir desse cálculo se definiu os meses com características de período chuvoso (aqueles meses com valor da precipitação acima da mediana) - junho, julho, agosto, setembro, outubro/2014 e março, maio, junho, julho/2015 - e os meses com características de período seco (aqueles meses com valor da precipitação abaixo da mediana) - novembro, dezembro /2014 e janeiro, fevereiro, abril, agosto, setembro, outubro, novembro $/ 2015$.

Morfologia externa. Os espécimes capturados foram classificados com base na morfologia externa das gônadas, para a determinação do estágio reprodutivo em: machos com testículos descendentes (TD), quando esses órgãos estavam localizados visivelmente na região escrotal; e machos com testículos não descendentes (TND), quando os testículos não estavam aparentes no escroto, estando situados na região abdominal (RACEY, 1988; GANNON \& WILLIG, 1992; EsBÉRARD, 2002).

Eutanásia dos animais. Foi aplicada inicialmente anestesia na região intraperitoneal do corpo, sendo a mesma constituída por pentobarbital sódico em uma concentração de $40 \mathrm{mg} / \mathrm{kg}^{-1}$. Na sequência, foi aplicada uma solução saturada constituída por cloreto de potássio (MoraIs et al., 2013b).

Coleta dos órgãos. Foram selecionados aleatoriamente 18 espécimes machos para as análises histomorfométricas dos órgãos. Após a eutanásia desses exemplares, os órgãos reprodutivos foram coletados, com auxílio de instrumentais de microdissecção. Foi realizada uma pequena incisão da pele e fáscias escrotal, na região inguinal, para expor o tecido subjacente até que os testículos fossem expostos e, consequentemente, removidos.

Análise histológica. Depois de removidos, todos os testículos foram fixados em formalina a $10 \%$ neutra tamponada (NBF) durante 24 horas e, posteriormente, conservados em álcool a $70 \%$ e glicerinado a $5 \%$. Na sequência, foram processados seguindo a técnica histológica de rotina (BEHMER et al., 1976; RIEDER \& SCHMIDT, 1987).

Esses órgãos foram impregnados em Paraplast e os blocos cortados no micrótomo em espessura de $4 \mu \mathrm{m}$. As lâminas histológicas produzidas foram coradas por Hematoxilina - Eosina (H.E.) e analisadas em microscópio óptico para verificar as microestruturas e tipos celulares existentes nos testículos.

Análises morfométricas. Nos testículos foram realizadas medidas da área de ocupação do compartimento tubular (AOCT) e área de ocupação do compartimento intertubular (AOCI), ambas mensuradas em percentual (\%) e fotografadas em aumento total de 100X, sendo consideradas 10 fotomicrografias por espécime (LIMA JUNIOR et al., 2014). 
Para contagem das células dos túbulos seminíferos foram considerados apenas os túbulos que continham todas as células da linhagem espermatogênica, assim no interior do compartimento tubular foram quantificados o número de células de Sertoli (NCS), o número de espermatócitos (NE) e o número de espermátides alongadas (NEA), enquanto que no compartimento intertubular foi quantificado o número total de células de Leydig (NCL). Para a contagem desses parâmetros, foram utilizadas fotomicrografias de 10 túbulos seminíferos diferentes e espaço intersticial, para cada espécime, em aumento total de 400X.

As lâminas histológicas foram fotografadas através do programa Motic Images Plus 2.0. Para isso, foi acoplada uma câmara ao microscópio óptico em conexão com um computador, projetando a lâmina no aumento desejado. As fotomicrografias foram submetidas às devidas mensurações, através do programa ImageJ.

Análise estatística. Os dados das amostras foram submetidos no programa SPSS (Statical Package of the Social Sciences, SPSS inc. Chicago, EUA) versão 15.0. Inicialmente foi utilizado o teste de Kolmogorov-Smirnov, o qual indicou que os dados da amostra não apresentavam uma distribuição normal. Por isso, foi utilizado o teste $\mathrm{U}$ de Mann-Whitney para avaliar os parâmetros morfométricos testiculares, de forma que os valores de $\mathrm{p}<0,05$ foram considerados estatisticamente significativos.

\section{RESULTADOS E DISCUSSÃO}

Foi coletado um total de 18 espécimes machos adultos de D.cinerea. Nos meses chuvosos, houve a ocorrência de indivíduos tanto com testículos descendentes (TD) $(n=04)$ quanto com testículos não descendentes (TND) $(n=07)$, enquanto que nos meses secos houve somente espécimes com TD $(\mathrm{n}=07)$. O mês com maior número de exemplares capturados foi julho/2014 ( $\mathrm{n}=05)$.

As análises histológicas evidenciaram que o compartimento tubular dos testículos dessa espécie apresentou espermatogônias, espermatócitos, espermátides e células de Sertoli ao longo dos meses chuvosos e secos, assim como independentemente da posição testicular. Verificou-se que o compartimento intertubular é constituído por células de Leydig e do tecido conjuntivo, bem como por vasos sanguíneos e linfáticos (Figs 1,2).

Os parâmetros histomorfométricos foram relacionados com o estágio reprodutivo (TD e TND) e com os meses chuvosos e secos. Em relação à posição testicular foram significativos e apresentaram as maiores médias: o NCS, o NE e o NEA (nos espécimes com TD) e a AOCI (nos espécimes com TND), enquanto a AOCT e o NCL não foram significativos. Os TD apresentaram maior variação relacionada aos parâmetros do compartimento tubular, enquanto que os TND demonstraram variação referente ao compartimento intertubular (Tab. I). Referente à precipitação, houve significância e apresentaram as maiores médias: a AOCI e a NCL (meses chuvosos) e o NCS, o NE e o NEA

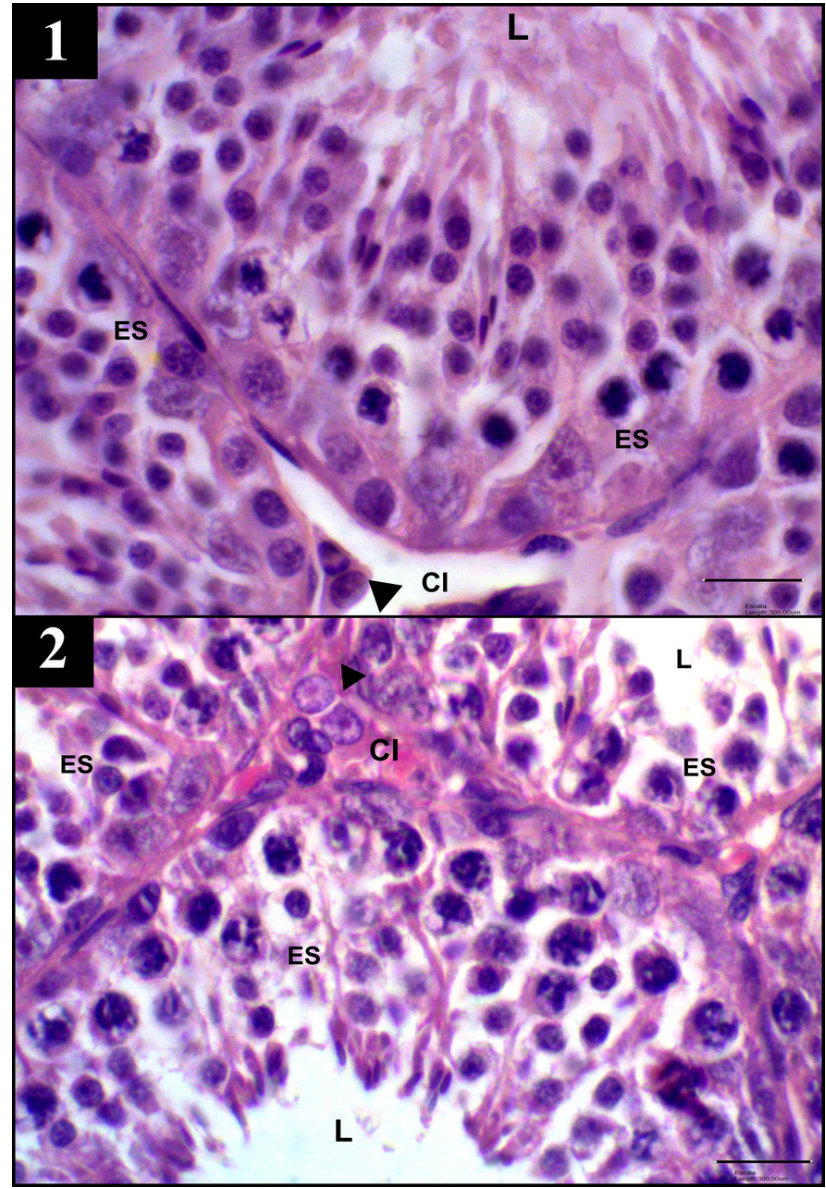

Figs 1, 2. Fotomicrografias do compartimento tubular e intertubular do testículo de Dermanura cinerea (Gervais, 1856) coletados em meses chuvosos (Fig. 1) e secos (Fig. 2). Observar o epitélio seminífero (ES) com células de Sertoli e da linhagem espermatogênica em diferentes estágios de maturação, e o lúmen (L) no compartimento tubular. Além das células de Leydig (cabeça de seta) distribuídas ao longo do compartimento intertubular (CI). Coloração: Hematoxilina - Eosina (H.E.). Aumento total: 1000 X. Barra de escala: $300 \mu \mathrm{m}$.

(meses secos), apenas a AOCT não apresentou diferença significativa (Tab. II).

As variações na precipitação influenciam diretamente a localização dos testículos de $D$. cinerea que apresenta ampla capacidade de ajustarem-se às condições ambientais. Esses órgãos podem migrar da região abdominal à escrotal em função da mudança de temperatura, sobretudo, em meses com índices pluviométricos elevados. Apesar disso, os espécimes tanto com TD quanto com TND apresentaram atividade reprodutiva, porém essa foi maior nos com TD, principalmente nos meses secos. Essas adaptações fisiológicas são de suma importância para manter a viabilidade da atividade androgênica, bem como da espermatogênese (JOLLY \& Blakshaw, 1988; Mello et al., 2009; Beguelini et al., 2013a,b,c,d; Morais et al., 2013a, 2014a,b; LIMA Junior et al., 2014).

Ao longo dos meses chuvosos os espécimes, sobretudo com TND, apresentaram um maior investimento na produção de testosterona, o que indica uma maior capacidade esteroidogênica nesse período. Isso foi constatado 
Tab. I. Relação da média e desvio-padrão, de cada um dos parâmetros histomorfométricos testiculares analisados, com a posição do testículo de Dermanura cinerea (Gervais, 1856) coletados em fragmento de Mata Atlântica do litoral sul de Pernambuco.

\begin{tabular}{cccc}
\hline \multirow{2}{*}{$\begin{array}{c}\text { Parâmetros } \\
\text { histomorfométricos }\end{array}$} & $\begin{array}{c}\text { Testículos não } \\
\text { descendentes } \\
\text { (TND) }\end{array}$ & $\begin{array}{c}\text { Testículos } \\
\text { descendentes } \\
\text { (TD) }\end{array}$ & \\
\cline { 2 - 4 } & $\begin{array}{c}\text { Média e } \\
\text { Desvio padrão }\end{array}$ & $\begin{array}{c}\text { Média e } \\
\text { Desvio padrão }\end{array}$ & Valor de p \\
\hline AOCT & $70,99 \pm 8,89$ & $73,08 \pm 9,44$ & 0,581 \\
AOCI & $7,76 \pm 4,96$ & $4,86 \pm 2,94$ & 0,004 \\
NCS & $9,47 \pm 3,85$ & $12,39 \pm 5,98$ & $<0,0001$ \\
NE & $36,50 \pm 18,86$ & $46,23 \pm 22,51$ & 0,001 \\
NEA & $38,13 \pm 22,23$ & $40,18 \pm 17,82$ & $<0,0001$ \\
NCL & $9,85 \pm 8,08$ & $7,06 \pm 6,88$ & 0,136 \\
\hline
\end{tabular}

pelo aumento do compartimento intertubular e das células de Leydig, as quais possuem maior predominância nesse compartimento, quando comparadas aos demais componentes existentes nesse espaço intersticial dos testículos (RUSSELL et al., 1990; ZiRKIN et al., 1994; MoraIs et al., 2013a, 2014a).

Nos meses de menor índice pluviométrico, os machos alocam mais energia para a maturação gonadal, produção e diferenciação celular que darão origem a espermatozoides viáveis para cópula. Isso foi expresso pela maior quantidade de células da linhagem espermatogênica e de Sertoli. Essas últimas possuem papel essencial na regulação e coordenação do processo de espermatogênese, visto que atuam na nutrição e sustentação dos diferentes tipos celulares que fazem parte desse processo fisiológico de elevado gasto energético (Nieschlag et al., 2010; Morais et al., 2013c; Farias et al., 2014).

Os diferentes tipos celulares da linhagem espermatogênica de $D$. cinerea são semelhantes ao que se tem relatado para Artibeus lituratus (Olfers, 1818) em área de Mata Atlântica no estado de Minas Gerais (DuARTE \& Talamoni, 2010; Notini et al., 2015) e Artibeus planirostris (Spix, 1823) no Sudeste do Brasil (BEGUELINI et al., 2013b). Ambas fazem parte da mesma subfamília (Stenodermatinae) que $D$. cinerea e também apresentaram atividade espermatogênica ao longo de todo ciclo reprodutivo anual. Esse comportamento possibilita a manutenção do sistema de acasalamento poligínico, no qual um macho dominante defende seu harém e impede a aproximação de outros machos para poder copular com diversas fêmeas da sua espécie (NowAK, 1994; ZorTÉA, 2003; BEGUELINI et al., 2013b).

A atividade espermatogênica de $D$. cinerea possui sincronia com o período de acasalamento das fêmeas. Isso ficou confirmado no estudo de LimA JUNIOR et al. (2018) com fêmeas dessa mesma espécie em um fragmento de Mata Atlântica do litoral sul de Pernambuco. Nessa investigação foi destacado que elas podem entrar em período gestacional durante meses secos e parir no final do período seco ou do início para a metade do período chuvoso, devido à maior disponibilidade de alimentos que subsidiem o período de lactação, assim como garantam a manutenção e sobrevivência
Tab. II. Relação da média e desvio-padrão de cada um dos parâmetros histomorfométricos testiculares analisados de Dermanura cinerea (Gervais, 1856), coletados durante meses chuvosos e secos, em fragmento de Mata Atlântica do litoral sul de Pernambuco.

\begin{tabular}{cccc}
\hline \multirow{2}{*}{$\begin{array}{c}\text { Parâmetros } \\
\text { histomorfométricos }\end{array}$} & $\begin{array}{c}\text { Média e } \\
\text { Desvio padrão }\end{array}$ & $\begin{array}{c}\text { Média e } \\
\text { Desvio padrão }\end{array}$ & Valor de p \\
\hline AOCT & $72,65 \pm 8,55$ & $71,67 \pm 10,33$ & 0,581 \\
AOCI & $6,60 \pm 4,45$ & $5,02 \pm 3,28$ & 0,004 \\
NCS & $9,98 \pm 4,56$ & $13,26 \pm 6,09$ & $<0,0001$ \\
NE & $37,42 \pm 17,69$ & $50,34 \pm 24,83$ & 0,001 \\
NEA & $36,15 \pm 20,21$ & $44,46 \pm 17,62$ & $<0,0001$ \\
NCL & $9,73 \pm 8,06$ & $6,28 \pm 5,56$ & 0,008 \\
\hline
\end{tabular}

da prole. $\mathrm{O}$ fato dos machos possuírem menor produção de esperma ao longo dos meses chuvosos, possivelmente indica um processo de recuperação do epitélio seminífero depois da ocorrência do período de acasalamento (SOARES et al., 2003; ZortÉA, 2003; OrtêNCIO FilHo et al., 2007; FARIAS et al., 2014; MoraIs et al., 2014a,b). Características semelhantes também foram relatadas para $A$. planirostris em área de Cerrado do noroeste de São Paulo, em que os maiores picos da espermatogênese ocorreram também em sincronia com a reprodução das fêmeas (BEGUELINı et al., 2013b).

Portanto, apesar de haver variações entre os parâmetros histomorfométricos testiculares averiguados dos espécimes com testículos descendentes e não descendentes, nos meses chuvosos e secos, conclui-se que $D$. cinerea apresentou atividade espermatogênica e esteroidogênica ao longo de todo o período considerado, cujos picos, em determinados meses, variam a partir da influência das condições ambientais em área de Mata Atlântica do Litoral sul de Pernambuco.

Agradecimentos. A Coordenação de Aperfeiçoamento de Pessoal de Nível Superior (CAPES) pela bolsa de mestrado concedida ao primeiro autor.

\section{REFERÊNCIAS BIBLIOGRÁFICAS}

Beguelini, M. R.; Goes, R. M.; Taboga, S. R. \& Morielle-Versute, E. 2013c. Two periods of total testicular regression are peculiar events of the annual reproductive cycle of the black Myotis bat, Myotis nigricans (Chiroptera: Vespertilionidae). Reproduction, Fertility and Development 26:834-846.

Beguelini, M. R.; Moreira, P. R. L.; Faria, K. C.; Marchesin, S. R. C. \& Morielle-Versute, E. 2009. Morphological Characterization of the Testicular Cells and Seminiferous Epithelium Cycle in Six Species of Neotropical Bats. Journal of Morphology 270:943-953.

Beguelini, M. R.; Puga, C. C. I.; Martins, F. F.; Betoli, A. H. S.; Taboga, S. R. \& Morielle-Versute, E. 2013a. Morphological Variation of Primary Reproductive Structures in Males of Five Families of Neotropical Bats. The Anatomical Record 296:156-167.

Beguelini, M. R.; Puga, C. C. I.; Taboga, S. R. \& Morielle-Versute, E. $2013 \mathrm{~b}$. Annual reproductive cycle of males of the flat-faced fruit-eating bat, Artibeus planirostris (Chiroptera: Phyllostomidae). General and Comparative Endocrinology 185:80-89.

Beguelini, M. R.; Taboga, S. R. \& Morielle-Versute, E. 2013d. Ultrastructural characteristics of the spermatogenesis during the four phases of the annual reproductive cycle of the black Myotis bat, Myotis 
nigricans (Chiroptera: Vespertilionidae). Microscopy Research and Technique 76:1035-1049.

Behmer, O. A.; Tolosa, E. M. C. \& Neto, A. G. F. 1976. Manual de Técnicas para Histologia Normal e Patológica. São Paulo, EDART - Ed. da Universidade de São Paulo. 331p.

Bredt, A.; Uieda, W. \& Magalhães, E. D. 1999. Morcegos cavernícolas da região do Distrito Federal, centro-oeste do Brasil (Mammalia, Chiroptera). Revista Brasileira de Zoologia 16(3):731-770.

Crichton, E. G. \& Krutzsch, P. H. 2000. Reproductive Biology of Bats. London, Academic Press. 510p.

DuARTE, A. P. G. \& TAlamoni, S. A. 2010. Reproduction of the large fruiteating bat Artibeus lituratus (Chiroptera: Phyllostomidae) in a Brazilian Atlantic forest area. Mammalian Biology 75:320-325.

EsbérARD, C. E. L. 2002. Composição da colônia e reprodução de Molossus rufus em refúgio no sudeste do Brasil (Mammalia, Molossidae). Revista Brasileira de Zoologia 19(4):1153-1160.

FABIÁN, M. E. \& MARQUES, R. V. 1989. Contribuição ao conhecimento da biologia reprodutiva de Molossus molossus (Pallas, 1766) (Chiroptera, Molossidae). Revista Brasileira de Zoologia 6(4):603-610.

Farias, T. O.; Notini, A. A.; Talamoni, S. A. \& Godinho, H. P. 2014. Testis Morphometry and Stages of the Seminiferous Epithelium Cycle in an Epididymal Sperm-storing Neotropical Vespertilionid, Myotis levis (Chiroptera). Anatomia, Histologia, Embryologia 44(5):361-369.

FranÇa, L. R. \& Russell, L. D. 1998. The testis of domestic mammals. In: Martinez-Garcia, F. \& Regardera, J. eds. Male Reproduction. A Multidisciplinary Overview. España, Churchill Communications, p.197-219.

Gannon, M. R. \& WiLLIG, M. R. 1992. Bat reproduction in the Luquillo Experimental Forest of Puerto Rico. Southwestern Naturalist 37(4):414-419.

GoMEs, M. N. \& UIEDA, W. 2004. Abrigos diurnos, composição de colônias, dimorfismo sexual e reprodução do morcego hematófago Desmodus rotundus (E. Geoffroy) (Chiroptera, Phyllostomidae) no Estado de São Paulo, Brasil. Revista Brasileira de Zoologia 21(3):629-638.

JoLLY, S. E. \& BLACKSHAW, A. W. 1988. Testicular migration, spermatogenesis, temperature regulation and environment of the sheath-tail bat, Taphozous georgianus. Journal of Reproduction and Fertility 84:447-455.

Lima Junior, N. B.; Arandas, M. J. G.; Marinho, K. S. N.; AgUiar JÚNior, F. C. A.; Pontes, A. R. M. \& SAntos, K. R. P. 2014. Histomorfometria testicular do morcego Phyllostomus discolor (Chiroptera: Phyllostomidae) em áreas de Mata Atlântica de Pernambuco. Brazilian Journal of Veterinary Research and Animal Science 51(3):263-270.

Lima Junior, N. B.; Arandas, M. J. G.; Silva, F. R.; Antonio, E. A.; Aguiar Júnior, F. C. A.; Teixeira, A. A. C.; Garcia, J. E. \& Santos, K. R. P. 2018. Analysis of the reproductive stage and ovarian histomorphometry of Dermanura cinerea (Chiroptera: Phyllostomidae) in an Atlantic Forest fragment of Pernambuco, northeastern Brazil. Pesquisa Veterinária Brasileira 38(1): 167-174.

Mello, M. A. R; Kalko, E. K. V. \& SiLVA, W. R. 2009. Ambient temperature is more important than food availability in explaining reproductive timing of the bat Sturnira lilium (Mammalia: Chiroptera) in a montane Atlantic forest. Canadian Journal of Zoology 87:239-245.

Mello, M. A. R.; Schittini, G. M.; Selig, P. \& Bergallo, H. G. 2004. Seasonal variantion in the diet of the Carollia perspicillata (Chiroptera: Phyllostomidae) in an Atlantic Forest area in southeastern Brazil. Mammalia 68(1):49-55.

Morais, D. B.; Barros, M. S.; Freitas, M. B. D.; Paula, T. A. R. \& MatTA, S. L. P. 2014a. Histomorphometric characterization of the intertubular compartment in the testes of the bat Sturnira lilium. Animal Reproduction Science 147(3-4): 180-186.

Morais, D. B.; Barros, M. S.; Paula, T. A. R.; Freitas, M. B. D.; Gomes, M. L. M. \& MatTA, S. L. P. 2014b. Evaluation of the Cell Population of the Seminiferous Epithelium and Spermatic Indexes of the Bat Sturnira lilium (Chiroptera: Phyllostomidae). Plos One 9(7):e101759.

Morais, D. B.; Cupertino, M. C.; Goulart, L. S.; Freitas, K. M.; Freitas, M. B. D.; Paula, T. A. R. \& MatTA, S. L. P. 2013c. Histomorphometric evaluation of the Molossus molossus (Chiroptera, Molossidae) testis: The tubular compartment and indices of sperm production. Animal Reproduction Science 140(3):268-278.
Morais, D. B.; Oliveira, L. C.; Cupertino, M. C.; Freitas, K. M.; Freitas, M. B. D.; Paula, T. A. R. \& MattA, S. L. P. 2013a. Organization and Seasonal Quantification of the Intertubular Compartment in the Bat Molossus molossus (Pallas, 1776) Testis. MicroscopyTechnique 76:94-101.

Morais, D. B.; Paula, T. A. R.; Barros, M. S.; Balarini, M. K.; Freitas, M. B. D. \& MAтTA, S. L. P. 2013b. Stages and duration of the seminiferous epithelium cycle in the bat Sturnira lilium. Journal of Anatomy 222:372-379.

Neuweiler, G. 2000. The biology of bats. Oxford, Oxford University Press. 310p.

Nieschlag, E.; Behre, H. M. \& Nieschlag, S. 2010. Physiology of testicular function. In: Weinbauer, G. F.; Luetjens, C. M.; Simioni, M. \& NiesChlaG, E. eds. Andrology: Male Reproductive Health and Dysfunction. Berlin, Springer, p. 15-16.

Notini, A. A.; Farias, T. O.; Talamoni, S.A. \& Godinho, H. P. 2015. Annual male reproductive activity and stages of the seminiferous epithelium cycle of the large fruit-eating Artibeus lituratus (Chiroptera: Phyllostomidae). Zoologia 32(3):195-200.

NowAK, R. M. 1994. Walker's Bats of the World. Baltimore, Jonhs Hopkins University Press. 287p.OrtêNCIO Filho, H.; ReIs, N. R.; PINTO, D. \& VieIRA, D. C. 2007. Aspectos reprodutivos de Artibeus lituratus (Phyllostomidae) em fragmentos florestais na região de Porto Rico, Paraná, Brasil. Chiroptera Neotropical 13(2):313-318.

RACEY, P. A. 1988. Reproductive assessment in bats. In: KunZ, T. H. ed. Ecological and behavioral methods for the study of bats. Washington, DC, Smithsonian Institution Press, p. 31-43.

Redondo, R. A. F.; Brina, L. P. S.; Silva, R. F.; Ditch-Field, A. D. \& Santos, F. R. 2008. Molecular systematics of the genus Artibeus (Chiroptera: Phyllostomidae). Molecular Phylogenetics and Evolution 49:44-58.

Reis, N. R.; Peracchi, A. L.; Pedro, W. A. \& Lima, I. P. eds. 2011. Mamíferos do Brasil. 2ed. Londrina, Editora da Universidade Estadual de Londrina. 439p. RiEder, N. \& SCHMIDT, K. 1987. Morphologische Arbeitsmethoden in der Biologie. Weinheim, Wiley-VCH, Gesellsch. $\mathrm{mbH} .232 \mathrm{p}$.

Rocha, P. A.; Mikalauskas, J. S.; Gouveia, S. F.; Silveira, V. V. B. \& Peracchi, A. L. 2010. Morcegos (Mammalia, Chiroptera) capturados no Campus da Universidade Federal de Sergipe, com oito novos registros para o estado. Biota Neotropica 10(3):183-188.

Russell, L. D.; Ettlin, R. A.; Sinha Hikim, A. P. \& ClegG, E. D. 1990. Mammalian spermatogenesis. In: Russell, L. D.; EtTlin, R. A.; SinHA HIKIM, A. P. \& CLEGG, E. D. eds. Histological and histopathological evaluation of the testis. Clearwater, Cache River Press, p. 1-40.

Scultori, C.; Dias, D. \& Peracchi, A. L. 2009. Mammalia, Chiroptera, Phyllostomidae, Artibeus cinereus: First record in the state of Paraná, Southern Brazil. Check List - Journal of Species Lists and Distribution 5(2):325-329.

Soares, M. C. F.; Urbinati, E. C. \& Senhorini, J. A. 2003. Variação Periódica da Triiodotironina (T3) Plasmática e sua Ação na Reprodução Induzida do Matrinxã, Brycon cephalus (Günther, 1869) em Cativeiro. Revista Brasileira de Zootecnia 32(6):1825-1834.

Solari, S.; Hoofer, S. R.; Larsen, P. A.; Brown, A. D.; Bull, R. J.; Guerrero, J. A.; Ortega, J.; Carrera, J. P.; Bradley, R. D. \& BAKER, R. J. 2009. Operational criteria for genetically defined species: analysis of the diversification of the small fruit-eating bats, Dermanura (Phyllostomidae: Stenodermatinae). Acta Chiropterologica 11(2):279-288.

Straube, F. C. \& BianConi, G. V. Sobre a grandeza e a unidade utilizada para estimar esforço de captura com utilização de redes-de-neblina. 2002. Chiroptera Neotropica 8(1-2):150-152.

Zirkin, B. R.; AwoniYi, C.; Griswold, M. D.; Russell, L. D. \& Sharpe, R. 1994. Is FSH required for adult spermatogenesis? Journal of Andrology 15:273-276.

ZortÉA, M. 2003. Reproductive patterns and feeding habits of three nectarivorous bats (Phyllostomidae: Glossophaginae) from the Brazilian Cerrado. Brazilian Journal of Biology 63(1):159-168. 